\title{
Certain Properties of a Class of Close-to-Convex Functions Related to Conic Domains
}

\author{
Wasim Ul-Haq and Shahid Mahmood \\ Department of Mathematics, Abdul Wali Khan University Mardan, Mardan 23200, Pakistan \\ Correspondence should be addressed to Wasim Ul-Haq; wasim474@hotmail.com \\ Received 30 September 2012; Revised 3 March 2013; Accepted 17 March 2013 \\ Academic Editor: Nikolaos Papageorgiou
}

Copyright (C) 2013 W. Ul-Haq and S. Mahmood. This is an open access article distributed under the Creative Commons Attribution License, which permits unrestricted use, distribution, and reproduction in any medium, provided the original work is properly cited.

We aim to define a new class of close-to-convex functions which is related to conic domains. Many interesting properties such as sufficiency criteria, inclusion results, and integral preserving properties are investigated here. Some interesting consequences of our results are also observed.

\section{Introduction}

Let $\mathscr{A}$ be the class of functions $f$

$$
f(z)=z+\sum_{n=2}^{\infty} a_{n} z^{n}
$$

which are analytic in the open unit disc $E=\{z \in \mathbb{C}:|z|<$ $1\}$. Let $f$ and $g$ be analytic in $E$, and we say that $f$ is subordinate to $g$, written as $f(z) \prec g(z)$ if there exists a Schwarz function $w$, which is analytic in $E$ with $w(0)=0$ and $|w(z)|<$ $1(z \in E)$, such that $f(z)=g(w(z))$. In particular, when $g$ is univalent, then the above subordination is equivalent to $f(0)=g(0)$ and $f(E) \subseteq g(E)$; see [1].

Kanas and Wisniowska $[2,3]$ introduced and studied the classes of $k$-uniformly convex functions denoted by $k-\mathscr{U} \mathscr{C V}$ and the corresponding class $k-\mathcal{S} \mathscr{T}$ related by the Alexandertype relation. Later, the class $k$-uniformly close-to-convex functions denoted by $k-\mathscr{U} \mathscr{K}$ defined as

$$
\begin{aligned}
k-\mathscr{U} \mathscr{K}= & \{f \in \mathscr{A}: \\
& \operatorname{Re}\left(\frac{z f^{\prime}(z)}{g(z)}\right)>k\left|\frac{z f^{\prime}(z)}{g(z)}-1\right|, \\
& g(z) \in k-\mathcal{S} \mathscr{T}, z \in E\}
\end{aligned}
$$

was considered by Acu [4]; for study details on these classes, we refer to [5-7]. All these above mentioned classes were generalized to the classes $\mathscr{S} \mathscr{D}(k, \delta), \mathscr{C} \mathscr{D}(k, \delta)$, and $\mathscr{K} \mathscr{D}(k, \beta, \delta)$ by Shams et al. [8] and Srivastava et al. [9], respectively. The classes $\mathscr{S} \mathscr{D}(k, \delta)$ and $\mathscr{C} \mathscr{D}(k, \delta)$ are defined as

$$
\begin{aligned}
\mathcal{S} \mathscr{D}(k, \delta)= & \{f \in \mathscr{A}: \\
& \operatorname{Re}\left(\frac{z f^{\prime}(z)}{f(z)}\right)>k\left|\frac{z f^{\prime}(z)}{f(z)}-1\right| \\
& +\delta, z \in E\}, \\
\mathscr{C} \mathscr{D}(k, \delta)=\left\{\begin{array}{l}
f \\
\qquad
\end{array}\right. & \operatorname{Re}\left(1+\frac{z f^{\prime \prime}(z)}{f^{\prime}(z)}\right)>k\left|\frac{z f^{\prime \prime}(z)}{f^{\prime}(z)}\right| \\
& +\delta, z \in E\},
\end{aligned}
$$

where $k \geq 0,0 \leq \beta, \delta<1$. The class $\mathscr{K} \mathscr{D}(k, \beta, \delta)$ known as $k$-uniformly close-to-convex functions of order $\beta$ type $\delta$ 
is the class of all those functions $f \in \mathscr{A}$ which satisfies the following condition:

$$
\begin{array}{r}
\operatorname{Re}\left(\frac{f^{\prime}(z)}{g^{\prime}(z)}\right) \geq k\left|\frac{f^{\prime}(z)}{g^{\prime}(z)}-1\right|+\beta, \\
(k \geq 0,0 \leq \beta, \delta<1 ; \quad z \in E),
\end{array}
$$

for some $g \in \mathscr{C} \mathscr{D}(k, \delta)$.

Motivated by the work of Noor et al. [10-13], we define the following.

Definition 1. Let $f \in \mathscr{A}$. Then, $f$ is in the class $\mathscr{T} \mathscr{D}(k, \alpha, \beta$, $\gamma, \delta)$ if and only if, for $\alpha \geq 0,0 \leq \beta, \gamma, \delta<1$,

$$
\begin{array}{r}
\operatorname{Re}(H(\alpha, \beta, \gamma ; f, g)) \geq k|H(\alpha, \beta, \gamma ; f, g)-1|, \\
(k \geq 0 ; z \in E),
\end{array}
$$

for some $g \in \mathscr{C} \mathscr{D}(k, \delta)$, where

$$
\begin{aligned}
H(\alpha, \beta, \gamma ; f, g)= & \frac{1-\alpha}{1-\beta}\left[\frac{f^{\prime}(z)}{g^{\prime}(z)}-\beta\right] \\
& +\frac{\alpha}{1-\gamma}\left[\frac{\left(z f^{\prime}(z)\right)^{\prime}}{g^{\prime}(z)}-\gamma\right] .
\end{aligned}
$$

\section{Special Cases}

(i) $(k, 0, \beta, \gamma, \delta)=\mathscr{K} \mathscr{D}(k, \beta, \delta)$; see [9].

(ii) $\mathscr{T} \mathscr{D}(1,0, \beta, \gamma, 0)=\mathscr{U} \mathscr{K}(\beta)$ and $\mathscr{T} \mathscr{D}(1,1, \beta, \gamma, 0)=$ $\mathscr{U Q}(\gamma)$, the classes of uniformly close-to-convex and quasiconvex functions introduced and investigated in [14].

(iii) $\mathscr{T} \mathscr{D}(0, \alpha, 0,0,0)=\mathbb{Q}_{\alpha}$, the class of alpha quasiconvex functions, introduced and studied in [11].

(iv) $\mathscr{T} \mathscr{D}(0,0 \beta, \gamma, \delta)=\mathscr{K}(\beta, \delta)$, the class of close-toconvex functions of order $\beta$ type $\delta$, [15].

(v) $\mathscr{T} \mathscr{D}(0,1, \beta, \gamma, \delta)=C^{*}(\gamma, \delta)$, the class of quasiconvex functions of order $\gamma$ type $\delta$, [16].

The conditions $k \geq 0, \alpha \geq 0,0 \leq \beta, \gamma, \delta<1$ on the parameters are assumed throughout the entire paper unless otherwise mentioned.

Geometric Interpretation. A function $f \in \mathscr{A}$ is in the class $\mathscr{T} \mathscr{D}(k, \alpha, \beta, \gamma, \delta)$ if and only if the functional $H(\alpha, \beta, \gamma, \delta ; f$, $g$ ) takes all the values in the conic domain $\Omega_{k}$ defined as follows:

$$
\Omega_{k}=\left\{u+\imath ; u>k \sqrt{(u-1)^{2}+v^{2}}\right\} .
$$

Extremal functions for these conic regions are denoted by $p_{k}(z)$, which are analytic in $E$ and map $E$ onto $\Omega_{k}$ such that $p_{k}(0)=1$ and $p_{k}^{\prime}(0)>1$. These functions are given as:

$$
p_{k}(z)=\left\{\begin{array}{lc}
\frac{1+z}{1-z}, & k=0, \\
1+\frac{2}{\pi^{2}}\left(\log \frac{1+\sqrt{z}}{1-\sqrt{z}}\right)^{2}, & k=1, \\
1+\frac{2}{1-k^{2}} \sinh ^{2}\left[\left(\frac{2}{\pi} \arccos k\right)\right. & \arctan h \sqrt{z}], \\
1+\frac{1}{k^{2}-1} \sin \left(\frac{\pi}{2 R(t)} \int_{0}^{u(z) / \sqrt{t}} \frac{1}{\sqrt{1-x^{2}} \sqrt{1-(t x)^{2}}} d x\right) \\
+\frac{1}{k^{2}-1}, & k>1,
\end{array}\right.
$$

where $u(z)=(z-\sqrt{t}) /(1-\sqrt{t z}), t \in(0,1), z \in E$, and $z$ is chosen such that $k=\cosh \left(\pi R^{\prime}(t) / 4 R(t)\right)$, where $R(t)$ is Legendre's complete elliptic integral of the first kind and $R^{\prime}(t)$ is complementary integral of $R(t)$; see $[2,3]$.

\section{Preliminaries Result}

We require the following results which are essential in our investigations.

Lemma 2 (see [17, page 70]). Let $h$ be convex function in $E$ and $q: E \mapsto C$ with $\operatorname{Re} q(z)>0, z \in E$. If $p$ is analytic in $E$ with $p(0)=h(0)$, then

$$
p(z)+q(z) z p^{\prime}(z) \prec h(z) \quad \text { implies } p(z) \prec h(z) .
$$

Lemma 3 (see [17, page 195]). Let $h$ be convex function in $E$ with $h(0)=0$ and $A \geq 0$. Suppose that $j \geq 4 / h^{\prime}(0)$ and that $B(z), C(z)$, and $D(z)$ are analytic in $E$ and satisfy

$$
\operatorname{Re} B(z) \geq A+|C(z)-1|-\operatorname{Re}(C(z)-1)+j D(z),
$$

for $z \in$ E. If $p$ is analytic in $E$ with $p(z)=a_{1} z+a_{2} z^{2}+a_{3} z^{3}+\cdots$ and the following subordination relation holds:

$$
A z^{2} p^{\prime \prime}(z)+B(z) z p^{\prime}(z)+C(z) p(z)+D(z) \prec h(z),
$$

then

$$
p(z) \prec h(z) .
$$

Lemma 4 (see [12]). If $f(z)<h(z)$ and $g(z)<h(z)$, then for $\alpha \in[0,1]$,

$$
(1-\alpha) f(z)+\alpha g(z) \prec h(z) .
$$

\section{Main Results}

First, we prove the following sufficiency criteria for the functions in the class $\mathscr{T} \mathscr{D}(k, \alpha, \beta, \gamma, \delta)$. 
Theorem 5. A function $f \in \mathscr{A}$ is said to be in the class $\mathscr{T} \mathscr{D}(k$, $\alpha, \beta, \gamma, \delta)$, if

$$
\sum_{n=2}^{\infty} \Phi_{n}(k ; \alpha, \beta, \gamma, \delta)<(1-\beta)(1-\gamma)
$$

where

$$
\begin{aligned}
& \Phi_{n}(k ; \alpha, \beta, \gamma, \delta) \\
& =(k+1)\left[(1-\alpha)(1-\gamma) n+\alpha(1-\beta) n^{2}\right]\left|a_{n}\right| \\
& +\left[(k+2-\beta)(1-\gamma)+\alpha(k+1)(\gamma-\beta) n\left|b_{n}\right|\right] .
\end{aligned}
$$

Proof. Let us assume that relation (6) holds. Now, it is sufficient to show that

$k|H(\alpha, \beta, \gamma, \delta ; f, g)-1|-\operatorname{Re}[H(\alpha, \beta, \gamma, \delta ; f, g)-1]<1$.

First, we consider

$$
\begin{aligned}
|H(\alpha, \beta, \gamma, \delta ; f, g)-1| & \mid \frac{1-\alpha}{1-\beta}\left[\frac{f^{\prime}(z)}{g^{\prime}(z)}-\beta\right] \\
& +\frac{\alpha}{1-\gamma}\left[\frac{\left(z f^{\prime}(z)\right)^{\prime}}{g^{\prime}(z)}-\gamma\right]-1 \mid \\
= & \mid \frac{1-\alpha}{1-\beta} \frac{f^{\prime}(z)}{g^{\prime}(z)}+\frac{\alpha}{1-\gamma} \frac{\left(z f^{\prime}(z)\right)^{\prime}}{g^{\prime}(z)} \\
& -\frac{(1-\alpha) \beta}{1-\beta}-\frac{\alpha \gamma}{1-\gamma}-1 \mid \\
= & \mid \frac{(1-\alpha)(1-\gamma) f^{\prime}(z)}{(1-\beta)(1-\gamma) g^{\prime}(z)} \\
& +\frac{\alpha(1-\beta)\left(z f^{\prime}(z)\right)^{\prime}}{(1-\beta)(1-\gamma) g^{\prime}(z)} \\
& -\frac{[(1-\gamma)+\alpha(\gamma-\beta)] g^{\prime}(z)}{(1-\beta)(1-\gamma) g^{\prime}(z)} \mid .
\end{aligned}
$$

Using (1) and the series $g(z)=z+\sum_{n=2}^{\infty} b_{n} z^{n}$ in (17), we have $|H(\alpha, \beta, \gamma, \delta ; f, g)-1|$

$$
\begin{aligned}
= & \mid \frac{(1-\alpha)(1-\gamma)\left(1+\sum_{n=2}^{\infty} n a_{n} z^{n-1}\right)}{(1-\beta)(1-\gamma)\left(1+\sum_{n=2}^{\infty} n b_{n} z^{n-1}\right)} \\
& +\frac{\alpha(1-\beta)\left(1+\sum_{n=2}^{\infty} n^{2} a_{n} z^{n-1}\right)}{(1-\beta)(1-\gamma)\left(1+\sum_{n=2}^{\infty} n b_{n} z^{n-1}\right)} \\
& -\frac{[(1-\gamma)+\alpha(\gamma-\beta)]\left(1+\sum_{n=2}^{\infty} n b_{n} z^{n-1}\right)}{(1-\beta)(1-\gamma)\left(1+\sum_{n=2}^{\infty} n b_{n} z^{n-1}\right)} \mid \\
= & \mid \frac{(1-\alpha)(1-\gamma)\left(\sum_{n=2}^{\infty} n a_{n} z^{n-1}\right)}{(1-\beta)(1-\gamma)\left(1+\sum_{n=2}^{\infty} n b_{n} z^{n-1}\right)}
\end{aligned}
$$

$$
+\frac{\alpha(1-\beta)\left(\sum_{n=2}^{\infty} n^{2} a_{n} z^{n-1}\right)}{(1-\beta)(1-\gamma)\left(1+\sum_{n=2}^{\infty} n b_{n} z^{n-1}\right)}
$$

$$
-\frac{[(1-\gamma)+\alpha(\gamma-\beta)]\left(\sum_{n=2}^{\infty} n b_{n} z^{n-1}\right)}{(1-\beta)(1-\gamma)\left(1+\sum_{n=2}^{\infty} n b_{n} z^{n-1}\right)} \mid
$$

$$
\leq \mid \frac{(1-\alpha)(1-\gamma)\left(\sum_{n=2}^{\infty} n\left|a_{n}\right||z|^{n-1}\right)}{(1-\beta)(1-\gamma)\left(1-\sum_{n=2}^{\infty} n\left|b_{n}\right||z|^{n-1}\right)}
$$

$$
+\frac{\alpha(1-\beta)\left(\sum_{n=2}^{\infty} n^{2}\left|a_{n}\right||z|^{n-1}\right)}{(1-\beta)(1-\gamma)\left(1-\sum_{n=2}^{\infty} n\left|b_{n}\right||z|^{n-1}\right)}
$$$$
+\frac{[(1-\gamma)+\alpha(\gamma-\beta)]\left(\sum_{n=2}^{\infty} n\left|b_{n}\right||z|^{n-1}\right)}{(1-\beta)(1-\gamma)\left(1-\sum_{n=2}^{\infty} n\left|b_{n}\right||z|^{n-1}\right)} \mid
$$$$
\leq \mid \frac{(1-\alpha)(1-\gamma)\left(\sum_{n=2}^{\infty} n\left|a_{n}\right|\right)}{(1-\beta)(1-\gamma)\left(1-\sum_{n=2}^{\infty} n\left|b_{n}\right|\right)}
$$

$$
\begin{aligned}
& +\frac{\alpha(1-\beta)\left(\sum_{n=2}^{\infty} n^{2}\left|a_{n}\right|\right)}{(1-\beta)(1-\gamma)\left(1-\sum_{n=2}^{\infty} n\left|b_{n}\right|\right)} \\
& +\frac{[(1-\gamma)+\alpha(\gamma-\beta)]\left(\sum_{n=2}^{\infty} n\left|b_{n}\right|\right)}{(1-\beta)(1-\gamma)\left(1-\sum_{n=2}^{\infty} n\left|b_{n}\right|\right)} \mid .
\end{aligned}
$$

Now,

$$
\begin{aligned}
k|H(\alpha, \beta, \gamma, \delta ; f, g)-1|-\operatorname{Re}[H(\alpha, \beta, \gamma, \delta ; f, g)-1] \\
\leq(k+1)|H(\alpha, \beta, \gamma, \delta ; f, g)-1| \\
\leq(k+1)\left[\frac{(1-\alpha)(1-\gamma)\left(\sum_{n=2}^{\infty} n\left|a_{n}\right|\right)}{(1-\beta)(1-\gamma)\left(1-\sum_{n=2}^{\infty} n\left|b_{n}\right|\right)}\right. \\
+\frac{\alpha(1-\beta)\left(\sum_{n=2}^{\infty} n^{2}\left|a_{n}\right|\right)}{(1-\beta)(1-\gamma)\left(1-\sum_{n=2}^{\infty} n\left|b_{n}\right|\right)} \\
\left.+\frac{[(1-\gamma)+\alpha(\gamma-\beta)]\left(\sum_{n=2}^{\infty} n\left|b_{n}\right|\right)}{(1-\beta)(1-\gamma)\left(1-\sum_{n=2}^{\infty} n\left|b_{n}\right|\right)}\right] .
\end{aligned}
$$


The last inequality is bounded above by 1 , if

$$
\begin{gathered}
(k+1)\left[(1-\alpha)(1-\gamma)\left(\sum_{n=2}^{\infty} n\left|a_{n}\right|\right)+\alpha(1-\beta)\left(\sum_{n=2}^{\infty} n^{2}\left|a_{n}\right|\right)\right. \\
\left.\quad+[(1-\gamma)+\alpha(\gamma-\beta)]\left(\sum_{n=2}^{\infty} n\left|b_{n}\right|\right)\right] \\
\leq(1-\beta)(1-\gamma)\left(1-\sum_{n=2}^{\infty} n\left|b_{n}\right|\right) .
\end{gathered}
$$

Hence,

$$
\sum_{n=2}^{\infty} \Phi_{n}(k ; \alpha, \beta, \gamma, \delta) \leq(1-\beta)(1-\gamma)
$$

where $\Phi_{n}(k ; \alpha, \beta, \gamma, \delta)$ is given by (15). This completes the proof.

When we take $\alpha=0, k=1$, and $g(z)=z$ in the above theorem, we obtain the following sufficient condition for the functions to be in the class $\mathscr{U} \mathscr{K}(\beta)$ which is proved in [14].

Corollary 6 (see [14]). A function $f \in A$ is said to be in the class $\mathcal{U} \mathscr{K}(\beta)$ if

$$
\sum_{n=2}^{\infty} n\left|a_{n}\right| \leq \frac{(1-\beta)}{2}
$$

Corollary 7 (see [14]). A function $f \in A$ is said to be in the class $\mathcal{U} Q(\gamma)$ if

$$
\sum_{n=2}^{\infty} n^{2}\left|a_{n}\right| \leq \frac{(1-\gamma)}{2}
$$

The above corollary is obtained when we take $\alpha=1, k=$ 1 , and $g(z)=z$ in Theorem 5 .

Theorem 8. Let $f \in \mathscr{T} \mathscr{D}(k, \alpha, \beta, \gamma, \delta)$ and $\alpha \geq 4(\beta /(1+3 \beta))$. Then, $f \in \mathscr{K} \mathscr{D}(k, 0, \delta)$.

Proof. Let

$$
\frac{f^{\prime}(z)}{g^{\prime}(z)}=p(z)
$$

where $p(z)$ is analytic and $p(0)=1$. Now differentiating (24), we have

$$
\frac{\left(z f^{\prime}(z)\right)^{\prime}}{g^{\prime}(z)}=z p^{\prime}(z)+p(z) \psi(z),
$$

where $\psi(z)=\left(z g^{\prime}(z)\right)^{\prime} / g^{\prime}(z)$. Using (24) and (25) in relation (6), we obtain

$$
\begin{aligned}
H(\alpha, \beta, \gamma, \delta ; f(z)) & \\
= & \frac{1-\alpha}{1-\beta}[p(z)-\beta]+\frac{\alpha}{1-\gamma}\left[z p^{\prime}(z)+p(z) \psi(z)-\gamma\right] \\
= & \frac{\alpha}{1-\gamma} z p^{\prime}(z)+\left[\frac{(1-\alpha)(1-\gamma)+\alpha(1-\beta) \psi(z)}{(1-\beta)(1-\gamma)}\right] p(z) \\
& -\frac{\beta(1-\alpha)(1-\gamma)+\alpha \gamma(1-\beta)}{(1-\beta)(1-\gamma)} \\
= & B(z) z p^{\prime}(z)+C(z) p(z)+D(z),
\end{aligned}
$$

where

$$
\begin{gathered}
B(z)=\frac{\alpha}{1-\gamma}, \quad C(z)=\frac{(1-\alpha)(1-\gamma)+\alpha(1-\beta) \psi(z)}{(1-\beta)(1-\gamma)}, \\
D(z)=-\frac{\beta(1-\alpha)(1-\gamma)+\alpha \gamma(1-\beta)}{(1-\beta)(1-\gamma)} .
\end{gathered}
$$

Now, since $f \in \mathscr{T} \mathscr{D}(k, \alpha, \beta, \gamma, \delta)$, we have

$$
B(z) z p^{\prime}(z)+C(z) p(z)+D(z) \prec p_{k}(z) .
$$

Replacing $p(z)$ by $p_{*}(z)=p(z)-1$ and $p_{k}(z)$ by $p_{k}^{*}(z)=$ $p_{k}(z)-1$, the above subordination is equivalent to

$$
B(z) z p_{*}^{\prime}(z)+C(z) p_{*}(z)+D_{*}(z) \prec p_{k}^{*}(z),
$$

where $D_{*}(z)=C(z)+D(z)-1$. Using Lemma 3 with $A=0$, we obtain

$$
p_{*}(z) \prec p_{k}^{*}(z)
$$

This implies that

$$
\frac{f^{\prime}(z)}{g^{\prime}(z)}=p(z) \prec p_{k}(z) .
$$

Hence, $f \in \mathscr{K} \mathscr{D}(k, 0, \delta)$. This completes the proof.

Corollary 9. Let $f \in \mathscr{T} \mathscr{D}(k, \alpha, 0,0,0)=\mathbb{Q}_{\alpha}$. Then, $f \in$ $\mathscr{K} \mathscr{D}(0,0,0)=\mathscr{K}$. That is, $\mathbb{Q}_{\alpha} \subset \mathscr{K}, \alpha \geq 0$.

The above result is well-known inclusion proved in [11].

For $f \in A$, consider the following integral operator defined by

$$
F(z)=I_{m}[f]=\frac{m+1}{z^{m}} \int_{0}^{z} t^{m-1} f(t) d t, \quad m=1,2,3 \ldots
$$

This operator was given by Bernardi [18] in 1969. In particular, the operator $I_{1}$ was considered by Libera [19]. Now let us prove the following. 
Theorem 10. Let $f \in \mathscr{T} \mathscr{D}(k, \alpha, \beta, \gamma, \delta)$. Then, $I_{m}[f] \in$ $\mathscr{K} \mathscr{D}(k, 0, \delta)$.

Proof. Let the function $g$ be such that (6) is satisfied. It can easily be seen that according to [4], the function $G=I_{m}[f] \epsilon$ $\mathscr{C} \mathscr{D}(k, \delta)$, and from (32), we deduce

$$
\begin{aligned}
& (1+m) f^{\prime}(z)=(1+m) F^{\prime}(z)+z F^{\prime \prime}(z), \\
& (1+m) g^{\prime}(z)=(1+m) G^{\prime}(z)+z G^{\prime \prime}(z) .
\end{aligned}
$$

If we let $p(z)=F^{\prime}(z) / G^{\prime}(z)$ and $q(z)=1 /(m+1+$ $\left.\left(z G^{\prime \prime}(z) / G^{\prime}\right)\right)$, then simple computations yield us

$$
\begin{aligned}
\frac{f^{\prime}(z)}{g^{\prime}(z)} & =\frac{(1+m) F^{\prime}(z)+z F^{\prime \prime}(z)}{(1+m) G^{\prime}(z)+z G^{\prime \prime}(z)} \\
& =\frac{z p(z) g^{\prime}(z)+z p(z) g^{\prime \prime}(z)+z g^{\prime}(z) p^{\prime}(z)}{(1+m) G^{\prime}(z)+z G^{\prime \prime}(z)} \\
& =p(z)+q(z) z p^{\prime}(z) .
\end{aligned}
$$

Let

$$
\frac{f^{\prime}(z)}{g^{\prime}(z)}=p(z)+z p^{\prime}(z) q(z)=h(z)
$$

where $h(z)$ is analytic and $p(0)=1$. From (36), we have

$$
\frac{\left(z f^{\prime}(z)\right)^{\prime}}{g^{\prime}(z)}=\psi(z) h(z)+z h^{\prime}(z),
$$

where $\psi(z)=\left(z g^{\prime}(z)\right)^{\prime} / g^{\prime}(z)$. Using (36) and (37) in (6), we have

$$
\begin{aligned}
H(\alpha, \beta, \gamma, \delta ; f(z)) & \\
= & \frac{1-\alpha}{1-\beta}[h(z)-\beta]+\frac{\alpha}{1-\gamma}\left[z h^{\prime}(z)+h(z) \psi(z)-\gamma\right] \\
= & \frac{\alpha}{1-\gamma} z h^{\prime}(z) \\
& +\left[\frac{(1-\alpha)(1-\gamma)+\alpha(1-\beta) \psi(z)}{(1-\beta)(1-\gamma)}\right] h(z) \\
& \quad-\frac{\beta(1-\alpha)(1-\gamma)+\alpha \gamma(1-\beta)}{(1-\beta)(1-\gamma)} \\
= & B(z) z h^{\prime}(z)+C(z) h(z)+D(z),
\end{aligned}
$$

where

$$
\begin{gathered}
B(z)=\frac{\alpha}{1-\gamma}, \quad C(z)=\frac{(1-\alpha)(1-\gamma)+\alpha(1-\beta) \psi(z)}{(1-\beta)(1-\gamma)}, \\
D(z)=-\frac{\beta(1-\alpha)(1-\gamma)+\alpha \gamma(1-\beta)}{(1-\beta)(1-\gamma)} .
\end{gathered}
$$

Now proceeding in the similar manner as in the proof of Theorem 5 and using Lemma 3 with $A=0$, we obtain

$$
\frac{f^{\prime}(z)}{g^{\prime}(z)}=h(z) \prec p_{k}(z) \text {. }
$$

From (36), it implies that

$$
p(z)+z p^{\prime}(z) q(z) \prec p_{k}(z) .
$$

By employing Lemma 2, we immediately obtain the desired result.

Theorem 11. For $\alpha>\alpha_{1} \geq 0$,

$$
\mathscr{T} \mathscr{D}(k, \alpha, \beta, \gamma, \delta) \subseteq \mathscr{T} \mathscr{D}\left(k, \alpha_{1}, \beta, \gamma, \delta\right) .
$$

Proof. Let $f \in \mathscr{T} \mathscr{D}(k, \alpha, \beta, \gamma, \delta)$. Then, consider

$$
\begin{aligned}
H\left(\alpha_{1}, \beta, \gamma, \delta ; f, g\right)= & \frac{1-\alpha_{1}}{1-\beta}\left[\frac{f^{\prime}(z)}{g^{\prime}(z)}-\beta\right] \\
& +\frac{\alpha_{1}}{1-\gamma}\left[\frac{\left(z f^{\prime}(z)\right)^{\prime}}{g^{\prime}(z)}-\gamma\right] .
\end{aligned}
$$

After some simple computations, we have

$$
\begin{aligned}
& H\left(\alpha_{1}, \beta, \gamma, \delta ; f, g\right) \\
& =\frac{1}{1-\beta}\left(1-\frac{\alpha_{1}}{\alpha}\right)\left[\frac{f^{\prime}(z)}{g^{\prime}(z)}-\beta\right] \\
& \quad+\frac{\alpha_{1}}{\alpha}\left[\frac{1-\alpha}{1-\beta}\left(\frac{f^{\prime}(z)}{g^{\prime}(z)}-\beta\right)+\frac{\alpha}{1-\gamma}\left(\frac{\left(z f^{\prime}(z)\right)^{\prime}}{g^{\prime}(z)}-\gamma\right)\right] \\
& =\left(1-\frac{\alpha_{1}}{\alpha}\right) H(0, \beta, \gamma, \delta ; f, g)+\frac{\alpha_{1}}{\alpha} H(\alpha, \beta, \gamma, \delta ; f, g) .
\end{aligned}
$$

Now, since $f \in \mathscr{T} \mathscr{D}(k, \alpha, \beta, \gamma, \delta)$, we have $H(\alpha, \beta, \gamma, \delta ; f, g) \prec$ $p_{k}(z)$. Also, Theorem 8 gives us that $H(0, \beta, \gamma, \delta ; f, g)<$ $p_{k}(z)$. The use of Lemma 4 leads us to the required relation; that is, $H\left(\alpha_{1}, \beta, \gamma, \delta ; f, g\right) \prec p_{k}(z)$. This completes the proof.

\section{Acknowledgment}

The authors would like to thank the reviewer of this paper for his/her valuable comments on the earlier version of this paper. They would also like to acknowledge Prof. Dr. Ehsan Ali, VC AWKUM, for the financial support on the publication of this article.

\section{References}

[1] A. W. Goodman, Univalent Functions. Vol. I, Polygonal Publishing House, Washington, DC, USA, 1983.

[2] S. Kanas and A. Wisniowska, "Conic regions and $k$-uniform convexity," Journal of Computational and Applied Mathematics, vol. 105, no. 1-2, pp. 327-336, 1999. 
[3] S. Kanas and A. Wisniowska, "Conic domains and starlike functions," Revue Roumaine de Mathématiques Pures et Appliquées, vol. 45, no. 4, pp. 647-657, 2000.

[4] M. Acu, "On a subclass of $n$-uniformly close to convex functions," General Mathematics, vol. 14, no. 1, pp. 55-64, 2006.

[5] E. Aqlan, J. M. Jahangiri, and S. R. Kulkarni, "New classes of $k$ uniformly convex and starlike functions," Tamkang Journal of Mathematics, vol. 35, no. 3, pp. 1-7, 2004.

[6] S. Kanas, "Alternative characterization of the class $k-U C V$ and related classes of univalent functions," Serdica. Mathematical Journal, vol. 25, no. 4, pp. 341-350, 1999.

[7] S. Kanas and H. M. Srivastava, "Linear operators associated with $k$-uniformly convex functions," Integral Transforms and Special Functions, vol. 9, no. 2, pp. 121-132, 2000.

[8] S. Shams, S. R. Kulkarni, and J. M. Jahangiri, "Classes of uniformly starlike and convex functions," International Journal of Mathematics and Mathematical Sciences, vol. 2004, no. 55, pp. 2959-2961, 2004.

[9] H. M. Srivastava, S.-H. Li, and H. Tang, "Certain classes of $k$ uniformly close-to-convex functions and other related functions defined by using the Dziok-Srivastava operator," Bulletin of Mathematical Analysis and Applications, vol. 1, no. 3, pp. 4963, 2009.

[10] K. I. Noor, "On a generalization of uniformly convex and related functions," Computers \& Mathematics with Applications, vol. 61, no. 1, pp. 117-125, 2011.

[11] K. I. Noor and F. M. Al-Oboudi, "Alpha-quasi-convex univalent functions," Caribbean Journal of Mathematics, vol. 3, pp. 1-8, 1984.

[12] K. I. Noor and S. N. Malik, "On generalized bounded Mocanu variation associated with conic domain," Mathematical and Computer Modelling, vol. 55, no. 3-4, pp. 844-852, 2012.

[13] K. I. Noor, M. Arif, and W. Ul-Haq, "On $k$-uniformly close-toconvex functions of complex order," Applied Mathematics and Computation, vol. 215, no. 2, pp. 629-635, 2009.

[14] K. G. Subramanian, T. V. Sudharsan, and H. Silverman, “On uniformly close-to-convex functions and uniformly quasiconvex functions," International Journal of Mathematics and Mathematical Sciences, vol. 2003, no. 48, pp. 3053-3058, 2003.

[15] R. J. Libera, “Some radius of convexity problems," Duke Mathematical Journal, vol. 31, pp. 143-158, 1964.

[16] K. I. Noor, "On quasi-convex functions and related topics," International Journal of Mathematics and Mathematical Sciences, vol. 2, pp. 241-258, 1987.

[17] S. S. Miller and P. T. Mocanu, Differential Subordinations: Theory and Applications, vol. 225 of Monographs and Textbooks in Pure and Applied Mathematics, Marcel Dekker, New York, NY, USA, 2000.

[18] S. D. Bernardi, "Convex and starlike univalent functions," Transactions of the American Mathematical Society, vol. 135, pp. 429446, 1969.

[19] R. J. Libera, "Some classes of regular univalent functions," Proceedings of the American Mathematical Society, vol. 16, pp. 755758, 1965. 


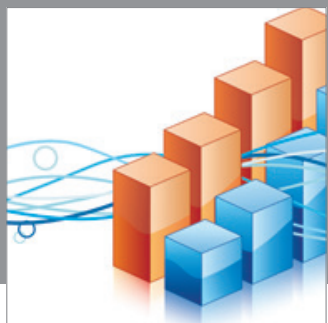

Advances in

Operations Research

mansans

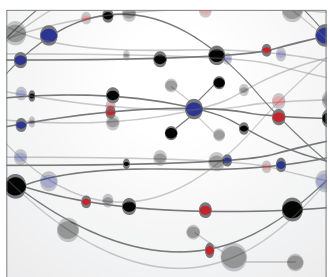

The Scientific World Journal
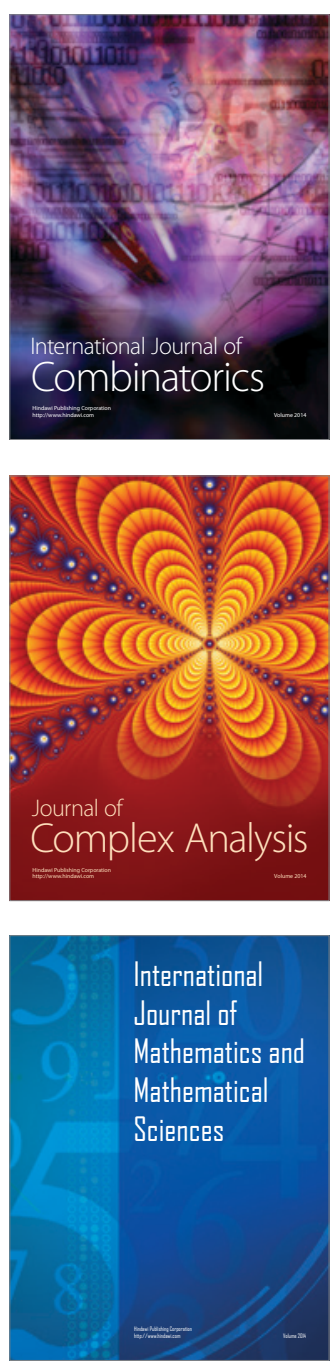
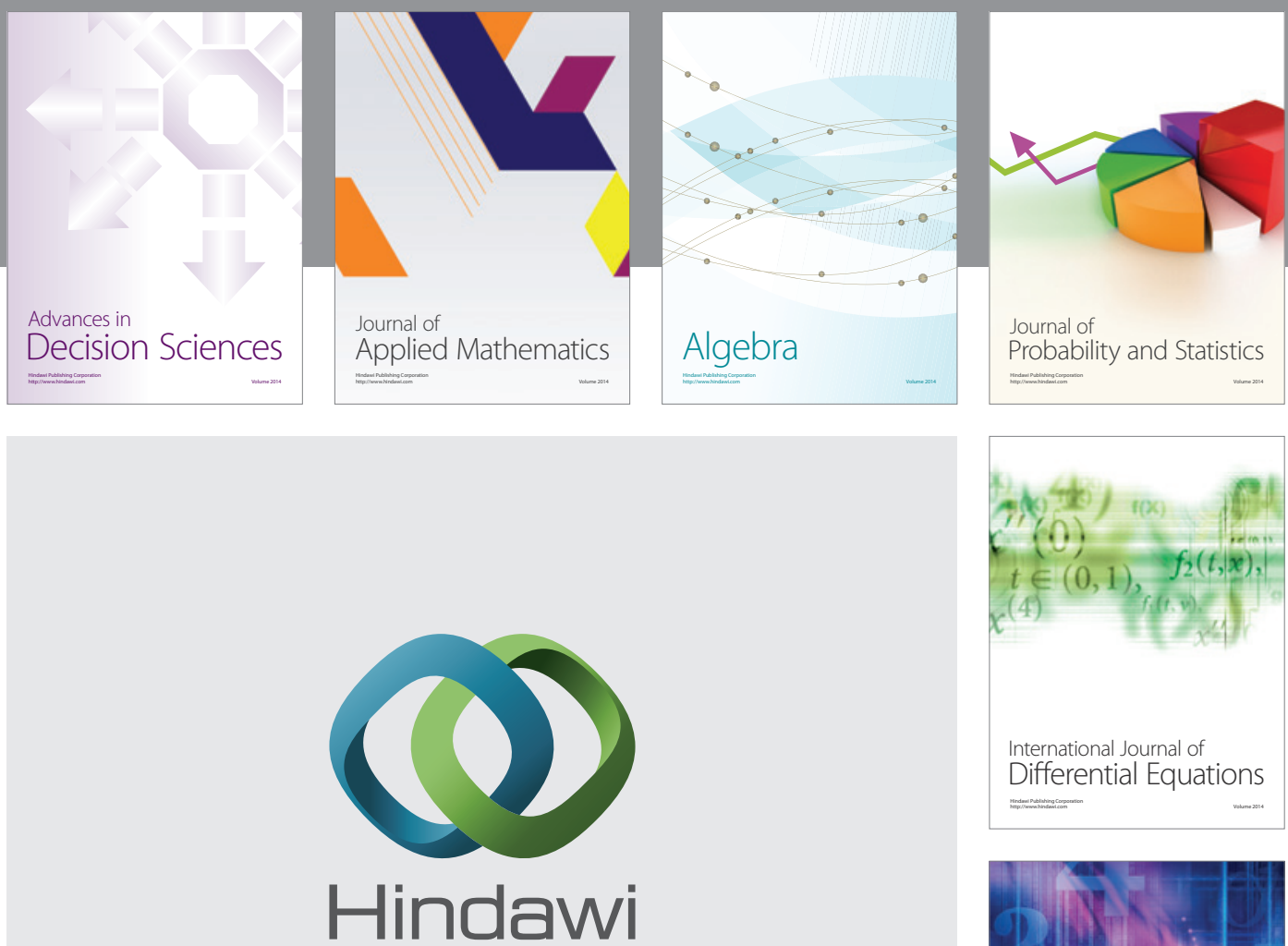

Submit your manuscripts at http://www.hindawi.com
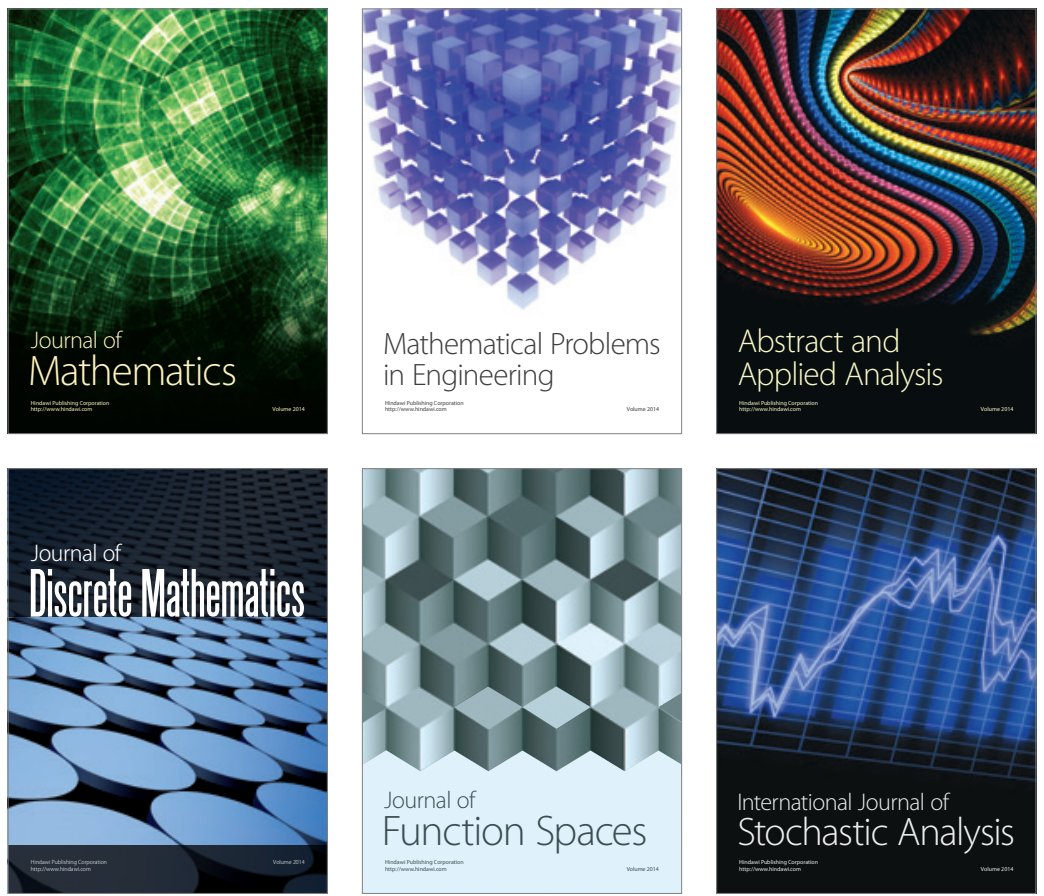

Journal of

Function Spaces

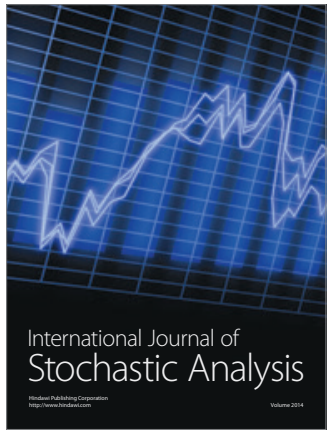

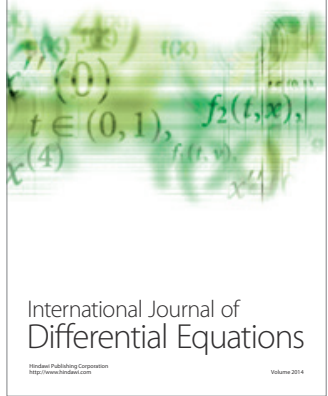
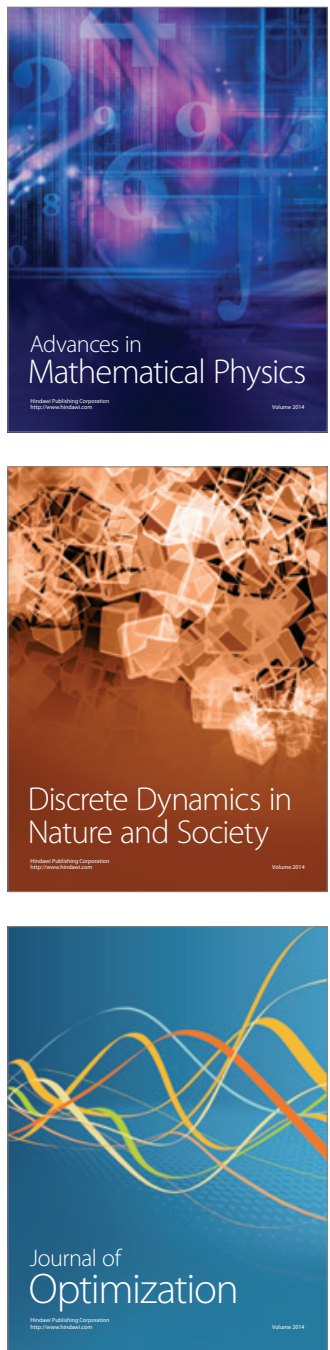